\title{
Qualidade de vida no trabalho em diferentes áreas de atuação profissional em um hospital
}

\author{
Quality of working life from the perspective of different groups \\ of professionals working in a maternity hospital
}

\author{
Sávio Ferreira Camargo (https://orcid.org/0000-0002-5165-1976) ${ }^{1,2}$ \\ Romanniny Hévillyn Silva Costa Almino (https://orcid.org/0000-0001-7159-0157) ${ }^{2,3}$ \\ Monique Pimentel Diógenes (https://orcid.org/0000-0001-9855-4682) ${ }^{2}$ \\ João Pedrosa de Oliveira Neto (https://orcid.org/0000-0002-9023-9618) ${ }^{2}$ \\ Ingrid Dantas Sampaio da Silva (https://orcid.org/0000-0001-5250-3994) ${ }^{2}$ \\ Leandro Cabral de Medeiros (https://orcid.org/0000-0003-2124-9713) ${ }^{2}$ \\ Kaio Graco Roque Dantas (https://orcid.org/0000-0003-4380-6314) ${ }^{2}$ \\ Juliana Dantas de Araújo Santos Camargo (https://orcid.org/0000-0001-8692-5706) ${ }^{2}$
}

\footnotetext{
${ }^{1}$ Programa de PósGraduação em Ciências da

Saúde, Universidade Federal do Rio Grande do Norte (UFRN). R. Gen. Gustavo Cordeiro de Faria 601, Ribeira. 59012-570 Natal RN Brasil.saviocamargo@ gmail.com

${ }^{2}$ Empresa Brasileira de Serviços Hospitalares, Maternidade Escola Januário Cicco. Natal RN

Brasil.

${ }^{3}$ Programa de Pós-

Graduação em Enfermagem, UFRN. Natal RN Brasil.
}

\begin{abstract}
The relationship between people and work has a direct impact on quality of life and health. The objective of this article is to compare perceived levels of Quality of Working Life (QWL) across three different groups of professionals. Cross-sectional study with a random sample of 172 hospital workers ( $37.9 \pm 10.3$ years; $73.8 \%$ women). Participants filled out the Quality of Working Life Assessment Inventory (QWL-AI). The data was analyzed using Anova and Tukey's test. Statistically significant differences were found between the groups in $36.4 \%$ of the items assessing work conditions, $35.7 \%$ of the items assessing professional growth and recognition $(p<0.05), 12.5 \%$ of the items assessing socioprofessional workplace relationships, $11.1 \%$ of the items assessing work organization, and 10\% of the items assessing link between work and social life. Overall, 21.7\% of the items showed significant differences between groups. We found significant differences in perceived QWL between the different groups, suggesting that QWL interventions should be tailored to the address the specific needs and demands of different sectors and departments to be effective.
\end{abstract}

Key words Quality of Life, Work, Health Promotion
Resumo A relação do homem com o trabalho pode impactar diretamente em sua qualidade de vida e condição de saúde. O objetivo deste artigo é comparar as condições de Qualidade de Vida no Trabalho (QVT) de três diferentes áreas de atuação profissional. Estudo transversal com amostra aleatória de 172 trabalhadores de um hospital (37,9 $\pm 10,3$ anos; $73,8 \%$ mulheres). Os participantes responderam ao inventário de avaliação de qualidade de vida no trabalho (IA_QVT). A Anova e o teste de Tukey foram utilizados para análise dos dados. 36,4\% dos itens que avaliaram condições de trabalho e 35,7\% dos itens sobre reconhecimento e crescimento profissional apresentaram diferenças entre os grupos $(p<0,05)$. Nos âmbitos de relações socioprofissionais, organização do trabalho e elo trabalho-vida social, os indices foram de $12,5 \%, 11,1 \%$ e 10,0\%, respectivamente. No geral, 21,7\% dos itens pesquisados apresentaram diferenças significativas entre os grupos. Foram encontradas diferenças importantes na percepção de QVT entre áreas distintas, sugerindo que um planejamento eficiente pode resultar em promoção da saúde caso atenda demandas setorizadas. Palavras-chave Qualidade de vida, Trabalho, Promoção da saúde 


\section{Introdução}

O processo de globalização pelo qual o mundo tem passado nas últimas décadas desencadeou transformações econômicas e sociais que alteraram a relação entre o homem e o seu trabalho ${ }^{1-4}$. $\mathrm{O}$ avanço do capitalismo resultou no crescimento das necessidades e desejos dos indivíduos, que buscam satisfazê-los por meio do trabalho ${ }^{4,5}$.

Com essa relação estreitada, passou-se a dar maior atenção ao bem-estar no trabalho ${ }^{6}$, que consiste no resultado das avaliações positivas sobre as diferentes características do ambiente laboral ${ }^{2}$, abrangendo componentes cognitivos, afetivos, motivacionais, psicossomáticos e comportamentais dos indivíduos ${ }^{6,7}$. Com as condições negativas surgem diversos sintomas institucionais como estresse, aumento de acidentes de trabalho e adoecimento, absenteísmo, queda da produtividade, entre outros ${ }^{8-11}$.

Qualidade de vida, segundo a Organização Mundial da Saúde, é definida como "[...] a percepção do indivíduo de sua posição na vida no contexto da cultura e sistema de valores nos quais ele vive e em relação aos seus objetivos, expectativas, padrões e preocupações [...]"»,12.

Promoção da saúde, conforme a Carta da Ottawa ${ }^{13}$, é o processo de capacitação da comunidade para atuar na melhoria de sua qualidade de vida e saúde. Para atingir um estado de completo bem-estar físico, mental e social, os indivíduos e grupos devem saber identificar aspirações, satisfazer necessidades e modificar favoravelmente o meio-ambiente.

A Qualidade de Vida no Trabalho (QVT) surge a partir do conceito inicial de qualidade de vida, sendo expressada por meio das representações globais (contexto organizacional) e específicas (situações de trabalho) que os trabalhadores constroem, indicando o predomínio de experiências de bem-estar no trabalho, de reconhecimentos institucional e coletivo, de possibilidade de crescimento profissional e de respeito às características individuais ${ }^{14}$.

Nesse cenário, a atenção à QVT surge como efetiva possibilidade de reduzir o mal-estar organizacional, resultando em repercussões positivas para a saúde dos trabalhadores ${ }^{10,15}$. A atenção à QVT precisa ser entendida como um campo para a promoção da saúde. Muito mais do que uma ferramenta administrativa ou estratégia para melhorar a produtividade em uma instituição, boas condições de QVT impactam de forma positiva no bem-estar dos profissionais, resultando em uma vida mais saudável ${ }^{16}$.
Intervenções assertivas de QVT são capazes de promover melhoria no bem-estar e na saúde dos trabalhadores ${ }^{7,9,11,15}$. Hipólito et al. ${ }^{17}$, em revisão sistemática sobre estudos de intervenção para a melhoria da QVT, evidenciam a importância da temática como fator benéfico para a saúde do trabalhador.

Contudo, diferentes classes profissionais que habitualmente compõem a força de trabalho em um hospital, como enfermeiros, técnicos de enfermagem e fisioterapeutas ${ }^{5,18-22}$, médicos ${ }^{22-25} \mathrm{e}$ corpo administrativo ${ }^{3,4,11,26,27}$, quando investigadas separadamente, apresentam diferenças quanto à auto-percepção de QVT.

Sob tais parâmetros, foi realizada esta pesquisa diagnóstica, cujas questões norteadoras do processo de investigação foram: "Como os integrantes de diferentes áreas profissionais percebem sua QVT?” e "Existe diferença significativa de percepção entre as áreas?”.

Dessa forma, nosso objetivo foi realizar um diagnóstico das condições de QVT em uma instituição hospitalar e analisar as diferenças de percepção entre três áreas profissionais: administrativa, assistencial e médica.

\section{Métodos}

\section{Delineamento, local e período do estudo}

Esta pesquisa é um estudo transversal realizado com os trabalhadores da Maternidade Escola Januário Cicco (MEJC), da Universidade Federal do Rio Grande do Norte, na cidade de Natal-RN, Brasil, entre fevereiro e dezembro de 2016.

\section{Participantes e amostragem}

Todos os servidores e empregados públicos com pelo menos dois meses de serviço no hospital foram elegíveis. A amostra foi do tipo probabilística e estratificada por área de atuação profissional. Os profissionais foram divididos em grupos/estratos, de forma que cada elemento da população pertencia somente a um estrato: área administrativa, área assistencial e área médica divisão usual no hospital para questões relativas a funcionários.

Para cálculo do tamanho amostral, utilizouse o universo de 513 trabalhadores e, com base no pré-teste, foi considerada a prevalência de $73 \%$ para a predominância de bem-estar no trabalho, erro amostral de $5 \%$ e nível de $95 \%$ de confiança. Em seguida, procedeu-se a uma amostra aleató- 
Foi utilizado como instrumento para coleta de dados, além de um questionário socioeconômico, o Inventário de Avaliação de Qualidade de Vida no Trabalho (IA_QVT), validado por Ferreira $^{29}$, instrumento de diagnóstico e monitoramento de QVT dentro de organizações corporativas que permite um eficaz mapeamento da percepção dos respondentes e a identificação de indicadores comportamentais, epidemiológicos e perceptivos de QVT.

Segundo Ferreira ${ }^{14}$, as representações de bem -estar e de mal-estar no trabalho têm origem em cinco fatores: Condições de Trabalho, Organização do Trabalho, Relações Socioprofissionais de Trabalho, Reconhecimento e Crescimento Profissional, e Elo Trabalho-Vida Social. A cultura organizacional em que se inserem os fatores tem importância estratégica para se interpretar a QVT, avaliada com base na aplicação do IA_QVT.

O inventário é composto por sessenta itens, distribuídos entre os diferentes fatores estruturadores. A avaliação sobre cada item é feita segundo uma gradação de 0 a 10. Para nortear a análise, é utilizada uma escala para avaliação das médias obtidas (Figura 1).

\section{Procedimentos}

Os participantes do estudo foram contatados para agendamento de preenchimento dos instrumentos de pesquisa disponibilizados como formulário eletrônico. Durante o procedimento, eles foram acompanhados por um entrevistador para padronização de entendimento, orientações e esclarecimento de dúvidas.

\section{Análise dos dados}

As variáveis socioeconômicas foram analisadas descritivamente por meio de percentuais. Os itens integrantes dos fatores estruturadores de QVT foram apresentados em média e desvio padrão (Média $\pm D P)$. Uma análise de variância de uma via (Anova) foi realizada para determinar se a QVT (médias dos fatores estruturadores) apresentava diferenças significativas entre as três áreas. A análise post hoc de Tukey foi realizada para indicação das áreas que apresentaram diferenças significativas. O nível de significância de $5 \%$ foi adotado para todas as análises ${ }^{28}$.

\section{Aspectos éticos}

Todos os participantes assinaram o termo de consentimento livre e esclarecido, de acordo com a Declaração de Helsinque. Para garantir o sigilo das informações, os questionários não continham identificação dos participantes e os pesquisadores que atuaram nas entrevistas não trabalharam na

\begin{tabular}{|c|c|c|c|c|c|c|c|c|c|}
\hline $0-0,9$ & $1-1,9$ & $2-2,9$ & $3-3,9$ & $\begin{array}{c}\text { Tendência } \\
\text { negativa } \\
4-4,9\end{array}$ & $\begin{array}{c}\text { Tendência } \\
\text { positiva } \\
5-5,9\end{array}$ & $6-6,9$ & $7-7,9$ & $8-8,9$ & $9-10$ \\
\hline Mal-es & intenso & \multicolumn{2}{|c|}{$\begin{array}{l}\text { Mal-estar } \\
\text { moderado }\end{array}$} & \multicolumn{2}{|c|}{ Zona de transição } & \multicolumn{2}{|c|}{$\begin{array}{l}\text { Bem-estar } \\
\text { moderado }\end{array}$} & \multicolumn{2}{|c|}{ Bem-estar intenso } \\
\hline \multicolumn{4}{|c|}{ Mal-estar dominante } & \multicolumn{2}{|c|}{ Zona de transição } & \multicolumn{4}{|c|}{ Bem-estar dominante } \\
\hline \multicolumn{4}{|c|}{$\begin{array}{l}\text { Resultado negativo que evidencia } \\
\text { a predominância de mal-estar no } \\
\text { trabalho } \\
\text { Risco de adoecimento }\end{array}$} & \multicolumn{2}{|c|}{$\begin{array}{l}\text { Resultado mediano. } \\
\text { Indicador de "situação- } \\
\text { limite". Coexistência de } \\
\text { mal-estar e bem-estar no } \\
\text { trabalho. } \\
\text { Estado de alerta }\end{array}$} & \multicolumn{4}{|c|}{$\begin{array}{l}\text { Resultado positivo que evidencia } \\
\text { a predominância de bem-estar no } \\
\text { trabalho. } \\
\text { Promoção de saúde }\end{array}$} \\
\hline
\end{tabular}

Figura 1. Cartografia psicométrica de interpretação dos resultados do IA_QVT. 
análise dos dados. A pesquisa foi aprovada pelo Comitê de Ética em Pesquisa do Hospital Universitário Onofre Lopes (HUOL/UFRN.)

\section{Resultados}

Para a caracterização da amostra, foram levantados dados amplos e alguns específicos sobre a realidade dos trabalhadores do hospital, conforme se verifica na Tabela 1.
A distribuição por sexo apresenta grande distinção entre as áreas estudadas. Enquanto a administrativa registra equilíbrio absoluto, a predominância do sexo feminino é verificada nas áreas assistencial e médica. Os entrevistados se enquadram com maior frequência nas faixas etárias de 30 a 39 e de 20 a 29 anos, tipificando uma população jovem. A maioria possui pósgraduação.

O tempo de serviço na instituição evidencia a recente contratação de grande contingente vin-

Tabela 1. Perfil socioeconômico da amostra.

\begin{tabular}{|c|c|c|c|}
\hline Variáveis & $\%$ & Variáveis & $\%$ \\
\hline Estado Civil & & Área profissional x Outro vínculo empregatício & \\
\hline Casado & 56 & Administrativa & \\
\hline Solteiro & 26 & Não & 93 \\
\hline Divorciado/separado & 10 & Sim & 7 \\
\hline União estável & 6 & Assistencial & \\
\hline Viúvo & 2 & Não & 46 \\
\hline Nível de Escolaridade & & Sim & 54 \\
\hline Pós-Graduação & 59 & Médica & \\
\hline Ensino Superior & 18 & Não & 13 \\
\hline Ensino Médio & 22 & Sim & 87 \\
\hline Ensino Fundamental & 1 & Área profissional x Cargo de chefia & \\
\hline Nível da Pós-Graduação & & Administrativa & \\
\hline Especialização & 86 & Não & 79 \\
\hline Mestrado & 11 & Sim & 21 \\
\hline Doutorado & 3 & Assistencial & \\
\hline Faixa Etária & & Não & 91 \\
\hline $20-29$ & 20 & Sim & 9 \\
\hline $30-39$ & 46 & Médica & \\
\hline $40-49$ & 16 & Não & 89 \\
\hline $50-59$ & 14 & Sim & 11 \\
\hline 60 ou mais & 4 & Área profissional x Carga horária semanal & \\
\hline Tempo de serviço na instituição & & Administrativa & \\
\hline Menos de 3 anos & 56 & entre 20 e $40 \mathrm{~h}$ & 89 \\
\hline 3 a 5 anos & 17 & acima de $40 \mathrm{~h}$ & 11 \\
\hline 6 a 10 anos & 3 & Assistencial & \\
\hline 11 a 16 anos & 10 & entre 20 e $40 \mathrm{~h}$ & 48 \\
\hline 17 a 30 anos & 7 & acima de $40 \mathrm{~h}$ & 52 \\
\hline 31 anos ou mais & 6 & Médica & \\
\hline Área profissional x Sexo & & entre 20 e $40 \mathrm{~h}$ & 19 \\
\hline Administrativa & & acima de $40 \mathrm{~h}$ & 81 \\
\hline Feminino & 50 & & \\
\hline Masculino & 50 & & \\
\hline \multicolumn{4}{|l|}{ Assistencial } \\
\hline Feminino & 81 & & \\
\hline Masculino & 19 & & \\
\hline \multicolumn{4}{|l|}{ Médica } \\
\hline Feminino & 71 & & \\
\hline Masculino & 29 & & \\
\hline
\end{tabular}


culado à Empresa Brasileira de Serviços Hospitalares (EBSERH), que, desde meados de 2014, é a responsável pela administração da instituição. A maioria dos entrevistados possui menos de três anos de atuação no hospital.

Quanto à questão de possuir outro vínculo profissional além do estabelecido com o hospital, a maioria nas áreas assistencial (54\%) e médica $(87 \%)$ respondeu positivamente. No corpo administrativo, mais de $90 \%$ possui exclusivamente o vínculo com a instituição pesquisada. $\mathrm{O}$ percentual de trabalhadores ocupantes de cargo de chefia em cada área indica que funcionários do setor administrativo têm maior participação nessas posições (22\%). Nas áreas médica e assistencial, o índice de ocupantes de cargos de chefia alcança aproximadamente metade do que é verificado na esfera administrativa: $11 \%$ e $9 \%$, respectivamente.

No levantamento da carga semanal de trabalho, registrou-se que na área administrativa apenas $11 \%$ trabalham em jornada superior a 40 horas semanais, padrão bastante diferenciado em relação às outras duas áreas: assistencial, pouco superior a $50 \%$; e médica, acima de $80 \%$.

\section{Interpretação dos Fatores Estruturadores de Qualidade de Vida no Trabalho}

Sob o ponto de vista dos parâmetros apresentados por Ferreira ${ }^{14}$, a avaliação encontrada nos cinco fatores estruturadores de QVT estão descritas na Tabela 2. Os fatores Condições de Trabalho e Organização do Trabalho foram os que apresentaram pior avaliação geral, ambos situados na zona de transição, que evidencia coexistência de mal-estar e bem-estar entre os trabalhadores.
As médias apresentadas na Tabela 2 representam o resultado geral, sem fragmentação das áreas profissionais. Após esse levantamento, foram realizados testes visando verificar a existência de diferenças estatisticamente significantes entre os grupos.

Em 21,7\% dos itens pesquisados, ocorreram diferenças significativas entre as áreas, considerando-se um nível de 5\% de significância. As diferenças entre os grupos foram evidenciadas em quatro dos onze itens componentes do fator Condições de Trabalho $(36,4 \%)$ e em cinco dos catorze itens componentes do fator Reconhecimento e Crescimento Profissional (35,7\%). Já nos fatores Relações Socioprofissionais, Organização do Trabalho e Elo Trabalho-Vida Social, os índices foram de 12,5\% (dois de dezesseis), 11,1\% (um de nove) e 10,0\% (um de dez), respectivamente.

O fator Condições de Trabalho apresentou diferenças significativas nos seguintes itens: $\mathrm{O}$ posto de trabalho é adequado para a realização das tarefas $(F[2 ; 169]=6,116, p=0,003)$; A temperatura ambiente é confortável $(\mathrm{F}[2 ; 169]=5,884$, $\mathrm{p}=0,003)$; O trabalho que realizo coloca em risco a minha segurança física $(F[2 ; 169]=14,964$, $\mathrm{p}=0,000)$; e O mobiliário existente no local de trabalho é adequado $(\mathrm{F}[2 ; 169]=11,079, \mathrm{p}=0,000)$.

O fator Organização do Trabalho apresentou diferenças significativas no item Falta tempo para ter pausa de descanso no trabalho $(F[2 ; 169]=6,432, p=0,002)$. O fator Relações Socioprofissionais de Trabalho apresentou diferenças significativas nos itens: É comum o conflito no ambiente de trabalho $(\mathrm{F}[2 ; 169]=3,309$, $\mathrm{p}=0,039)$ e Meus colegas de trabalho demonstram disposição em me ajudar $(\mathrm{F}[2 ; 169]=3,581$, $\mathrm{p}=0,030)$.

Tabela 2. Média geral e itens destacados dos fatores estruturadores de QVT.

\begin{tabular}{|c|c|c|}
\hline Fator & Média $\pm \mathrm{DP}$ & Itens de melhor e de pior avaliação \\
\hline $\begin{array}{l}\text { Condições de } \\
\text { trabalho }\end{array}$ & $5,60 \pm 2,86$ & $\begin{array}{l}\text { O nível de iluminação é suficiente para executar as atividades }(6,93) \\
\text { O espaço físico é satisfatório }(4,31)\end{array}$ \\
\hline $\begin{array}{l}\text { Organização do } \\
\text { trabalho }\end{array}$ & $5,27 \pm 2,88$ & $\begin{array}{l}\text { Posso executar o meu trabalho sem pressão }(7,12) \\
\text { As tarefas são repetitivas }(3,60)\end{array}$ \\
\hline $\begin{array}{l}\text { Relações } \\
\text { socioprofissionais } \\
\text { de trabalho }\end{array}$ & $7,89 \pm 2,18$ & $\begin{array}{l}\text { Minhas relações de trabalho com os colegas são harmoniosas }(9,07) \\
\text { A comunicação entre funcionários é insatisfatória }(6,15)\end{array}$ \\
\hline $\begin{array}{l}\text { Reconhecimento } \\
\text { e crescimento } \\
\text { profissional }\end{array}$ & $6,20 \pm 2,92$ & $\begin{array}{l}\text { A prática do reconhecimento contribui para a minha realização } \\
\text { profissional }(7,89) \\
\text { O reconhecimento do trabalho individual é uma prática efetiva } \\
(4,75)\end{array}$ \\
\hline $\begin{array}{l}\text { Elo trabalho-vida } \\
\text { social }\end{array}$ & $8,04 \pm 2,01$ & $\begin{array}{l}\text { O trabalho que faço é útil para a sociedade }(9,41) \\
\text { A sociedade reconhece a importância do meu trabalho }(6,70)\end{array}$ \\
\hline
\end{tabular}


Tabela 3. Medidas descritivas dos itens com diferenças significativas entre as áreas profissionais.

\begin{tabular}{|c|c|c|c|}
\hline \multirow[b]{2}{*}{ Fator/Item } & \multicolumn{3}{|c|}{ Média $\pm D P$} \\
\hline & $\begin{array}{l}\text { Administrativa } \\
\mathrm{n}=\mathbf{2 8}\end{array}$ & $\begin{array}{c}\text { Assistencial } \\
\mathrm{n}=106\end{array}$ & $\begin{array}{c}\text { Médica } \\
\mathrm{n}=38\end{array}$ \\
\hline \multicolumn{4}{|l|}{ Condições de Trabalho } \\
\hline O posto de trabalho é adequado para a realização das tarefas & $7,07 \pm 2,64^{\mathrm{a}}$ & $5,38 \pm 2,88^{\mathrm{a}, \mathrm{b}}$ & $6,66 \pm 2,13^{b}$ \\
\hline A temperatura ambiente é confortável & $7,64 \pm 2,21^{\mathrm{a}}$ & $5,89 \pm 3,20^{\mathrm{a}, \mathrm{b}}$ & $7,29 \pm 2,50^{\mathrm{b}}$ \\
\hline O trabalho que realizo coloca em risco a minha segurança física & $7,50 \pm 2,83^{\mathrm{a}, \mathrm{c}}$ & $4,33 \pm 2,97^{\mathrm{a}}$ & $3,82 \pm 3,07^{c}$ \\
\hline O mobiliário existente no local de trabalho é adequado & $7,32 \pm 2,36^{\mathrm{a}}$ & $5,07 \pm 2,87^{\mathrm{a}, \mathrm{b}}$ & $6,82 \pm 2,39^{\mathrm{b}}$ \\
\hline \multicolumn{4}{|l|}{ Organização do Trabalho } \\
\hline Falta tempo para ter pausa de descanso no trabalho & $6,82 \pm 3,26^{\mathrm{a}, \mathrm{c}}$ & $4,45 \pm 3,19^{\mathrm{a}}$ & $4,47 \pm 3,15^{\mathrm{c}}$ \\
\hline \multicolumn{4}{|l|}{ Relações Socioprofissionais de Trabalho } \\
\hline É comum o conflito no ambiente de trabalho & $7,71 \pm 2,99^{\mathrm{a}}$ & $6,07 \pm 3,09^{\mathrm{a}}$ & $6,47 \pm 2,84$ \\
\hline Meus colegas de trabalho demonstram disposição em me ajudar & $9,29 \pm 1,05^{\mathrm{a}}$ & $8,54 \pm 1,63^{\mathrm{a}}$ & $8,97 \pm 1,00$ \\
\hline \multicolumn{4}{|l|}{ Reconhecimento e Crescimento Profissional } \\
\hline $\begin{array}{l}\text { A prática do reconhecimento contribui para a minha realização } \\
\text { profissional }\end{array}$ & $6,93 \pm 2,73^{c}$ & $7,92 \pm 2,40$ & $8,53 \pm 1,83^{c}$ \\
\hline A MEJC oferece oportunidade de crescimento profissional & $4,21 \pm 3,39^{c}$ & $5,32 \pm 3,08$ & $6,50 \pm 2,02^{c}$ \\
\hline $\begin{array}{l}\text { O reconhecimento do trabalho coletivo é uma prática efetiva na } \\
\text { MEJC }\end{array}$ & $5,29 \pm 3,53$ & $5,19 \pm 2,93^{\mathrm{b}}$ & $6,84 \pm 1,85^{\mathrm{b}}$ \\
\hline $\begin{array}{l}\text { As oportunidades de crescimento profissional são iguais para } \\
\text { todos }\end{array}$ & $3,75 \pm 3,88^{c}$ & $5,26 \pm 3,31$ & $6,00 \pm 2,84^{\mathrm{c}}$ \\
\hline $\begin{array}{l}\text { O reconhecimento do trabalho individual é uma prática efetiva } \\
\text { na MEJC }\end{array}$ & $4,50 \pm 3,66$ & $4,36 \pm 3,01^{\mathrm{b}}$ & $6,03 \pm 2,55^{b}$ \\
\hline \multicolumn{4}{|l|}{ Elo Trabalho-Vida Social } \\
\hline O trabalho que faço é útil para a sociedade & $8,82 \pm 1,68^{\mathrm{a}, \mathrm{c}}$ & $9,48 \pm 0,80^{\mathrm{a}}$ & $9,63 \pm 0,88^{\mathrm{c}}$ \\
\hline
\end{tabular}

O fator Reconhecimento e Crescimento Profissional apresentou diferenças significativas nos itens: A prática do reconhecimento contribui para a minha realização profissional $(\mathrm{F}[2 ; 169]=3,749$, $\mathrm{p}=0,026)$; A MEJC oferece oportunidade de crescimento profissional $(\mathrm{F}[2 ; 169]=4,994, \mathrm{p}=0,008)$; O reconhecimento do trabalho coletivo é uma prática efetiva na MEJC $(\mathrm{F}[2 ; 169]=4,908$, $\mathrm{p}=0,008)$; As oportunidades de crescimento profissional são iguais para todos $(\mathrm{F}[2 ; 169]=3,800$, $\mathrm{p}=0,024)$; e $\mathrm{O}$ reconhecimento do trabalho individual é uma prática efetiva na MEJC $(\mathrm{F}[2 ; 169]=4,338, \mathrm{p}=0,015)$.

O fator Elo Trabalho-Vida Social apresentou diferenças significativas no item $\mathrm{O}$ trabalho que faço é útil para a sociedade $(\mathrm{F}[2 ; 169]=5,965$, $\mathrm{p}=0,003)$. A Tabela 3 apresenta as medidas descritivas desses itens.

A Tabela 4 apresenta os resultados da análise post hoc de Tukey, especificando a diferença média e os intervalos de confiança (IC) de 95\% para essa diferença, assim como os valores de significância estatística (valor p).
A diferença média de avaliação entre as áreas foi evidenciada fundamentalmente tendo do lado inferior as áreas assistencial ou administrativa e do lado superior ora a área médica, ora a área administrativa.

\section{Discussão}

Este estudo evidenciou padrões diferenciados no diagnóstico de QVT em uma comparação entre diferentes áreas profissionais. $\mathrm{O}$ foco da discussão é explorar as principais diferenças, ressaltando a importância da não generalização de intervenções de QVT no local de trabalho.

É preciso atentar para as necessidades dos trabalhadores, identificando, principalmente, situações de mal-estar, e implementando intervensões no âmbito da QVT capazes de melhorar o bem-estar dos trabalhadores e, consequentemente, seu estado geral de saúde ${ }^{1,4,7}$.

Entre os fatores estruturadores de QVT, os dois que apresentaram maiores diferenças entre 
Tabela 4. Teste de Tukey para os fatores estruturadores de QVT.

\begin{tabular}{|c|c|c|c|c|}
\hline Fator/Itens & Áreas & $\begin{array}{l}\text { Diferença } \\
\text { média }\end{array}$ & IC95\% & Valor $\mathbf{p}$ \\
\hline \multicolumn{5}{|l|}{ Condições de Trabalho } \\
\hline \multirow{2}{*}{$\begin{array}{l}\text { O posto de trabalho é adequado para a realização das } \\
\text { tarefas }\end{array}$} & ADM - ASS & 1,694 & $(0,339 ; 3,049)$ & $0,010^{(1)}$ \\
\hline & MED - ASS & 1,281 & $(0,075 ; 2,486)$ & $0,034^{(1)}$ \\
\hline \multirow[t]{2}{*}{ A temperatura ambiente é confortável } & ADM - ASS & 1,756 & $(0,291 ; 3,221)$ & $0,014^{(1)}$ \\
\hline & MED - ASS & 1,403 & $(0,099 ; 2,706)$ & $0,032^{(1)}$ \\
\hline \multirow{2}{*}{$\begin{array}{l}\text { O trabalho que realizo coloca em risco a minha } \\
\text { segurança física }\end{array}$} & ADM - ASS & 3,170 & $(1,679 ; 4,661)$ & $0,000^{(1)}$ \\
\hline & ADM - MED & 3,684 & $(1,937 ; 5,432)$ & $0,000^{(1)}$ \\
\hline \multirow{2}{*}{$\begin{array}{l}\text { O mobiliário existente no local de trabalho é } \\
\text { adequado }\end{array}$} & ADM - ASS & 2,255 & $(0,901 ; 3,610)$ & $0,000^{(1)}$ \\
\hline & MED - ASS & 1,750 & $(0,544 ; 2,955)$ & $0,002^{(1)}$ \\
\hline \multicolumn{5}{|l|}{ Organização do Trabalho } \\
\hline \multirow[t]{2}{*}{ Falta tempo para ter pausa de descanso no trabalho } & ADM - ASS & 2,369 & $(0,766 ; 3,971)$ & $0,002^{(1)}$ \\
\hline & ADM - MED & 2,348 & $(0,469 ; 4,226)$ & $0,010^{(1)}$ \\
\hline \multicolumn{5}{|l|}{ Relações Socioprofissionais de Trabalho } \\
\hline É comum o conflito no ambiente de trabalho & ADM - ASS & 1,648 & $(0,132 ; 3,165)$ & $0,030^{(1)}$ \\
\hline $\begin{array}{l}\text { Meus colegas de trabalho demonstram disposição em } \\
\text { me ajudar }\end{array}$ & ADM - ASS & 0,748 & $(0,028 ; 1,468)$ & $0,040^{(1)}$ \\
\hline \multicolumn{5}{|l|}{ Reconhecimento e Crescimento Profissional } \\
\hline $\begin{array}{l}\text { A prática do reconhecimento contribui para a minha } \\
\text { realização profissional }\end{array}$ & MED - ADM & 1,598 & $(0,215 ; 2,98)$ & $0,0019^{(1)}$ \\
\hline $\begin{array}{l}\text { A MEJC oferece oportunidade de crescimento } \\
\text { profissional }\end{array}$ & MED - ADM & 2,286 & $(0,558 ; 4,014)$ & $0,006^{(1)}$ \\
\hline $\begin{array}{l}\text { O reconhecimento do trabalho coletivo é uma prática } \\
\text { efetiva na MEJC }\end{array}$ & MED - ASS & 1,653 & $(0,384 ; 2,923)$ & $0,007^{(1)}$ \\
\hline $\begin{array}{l}\text { As oportunidades de crescimento profissional são } \\
\text { iguais para todos }\end{array}$ & MED - ADM & 2,250 & $(0,297 ; 4,203)$ & $0,019^{(1)}$ \\
\hline $\begin{array}{l}\text { O reconhecimento do trabalho individual é uma } \\
\text { prática efetiva na MEJC }\end{array}$ & MED - ASS & 1,668 & $(0,311 ; 3,025)$ & $0,011^{(1)}$ \\
\hline \multicolumn{5}{|l|}{ Elo Trabalho-Vida Social } \\
\hline \multirow[t]{2}{*}{ O trabalho que faço é útil para a sociedade } & ASS - ADM & 0,660 & $(0,154 ; 1,166)$ & $0,007^{(1)}$ \\
\hline & MED - ADM & 0,810 & $(0,217 ; 1,403)$ & $0,004^{(1)}$ \\
\hline
\end{tabular}

${ }^{1}$ Significativo a 5\%. ADM: Administrativa; ASS: Assistencial; MED: Médica.

as três áreas estudadas foram Condições de Trabalho, com 36,4\%, e Reconhecimento e Crescimento Profissional, com $35,7 \%$ dos itens com diferença significativa entre as áreas.

O fator Condições de Trabalho é tido como um dos principais pilares de investigação dos contextos laborais e, quando consideradas inadequadas, tais condições podem conduzir a representações de mal-estar no trabalho ${ }^{9,30}$. Um ambiente de trabalho seguro e saudável para o exercício das atividades favorece uma boa saúde física e mental para os trabalhadores ${ }^{1,30}$.

O fornecimento de suporte organizacional consiste em requisito indispensável para a promoção da QVT, reduzindo riscos à segurança e à saúde do trabalhador e, sobretudo, facilitando o processo de adaptação às exigências do trabalho $^{26}$. No caso estudado, os trabalhadores da área assistencial necessitam de uma intervenção diferenciada no referido fator, pois apresentaram nível de satisfação com as condições de trabalho significativamente menor que aqueles das áreas médica e administrativa.

A existência de momentos para uma pausa de descanso durante o trabalho foi, na área administrativa $(6,82 \pm 3,26 ; p=0,002)$, significativamente melhor avaliada do que nas áreas assistencial $(4,45 \pm 3,19 ; \mathrm{p}=0,002)$ e médica $(4,47 \pm 3,15$; $\mathrm{p}=0,010)$. Esse aspecto pode ser potencializado pelo duplo ou triplo vínculo empregatício, co- 
mum entre profissionais da assistência à saúde, $\mathrm{o}$ que pode representar impacto negativo na qualidade de vida ${ }^{19-21,30}$.

Daubermann e Tonete ${ }^{20}$ abordam essa questão quando constatam associação entre a sobrecarga de trabalho resultante de vínculos duplos ou triplos e situações adversas relatadas por profissionais de saúde. E afirmam, ainda, que a adoção do modelo curativo de QVT em detrimento da prevenção e promoção da saúde também representa um elemento desfavorável ${ }^{20}$.

Sobre o fator Relações Socioprofissionais, a análise deve partir da premissa de que o contexto influencia as emoções, o humor e o afeto do trabalhador, o que pode facilitar ou dificultar as interações sociais e o desenvolvimento de clima propício ao bem-estar no trabalho ${ }^{3}$.

Nesse fator, a ocorrência de conflitos foi significativamente maior e a disposição dos colegas em auxiliar no serviço foi menor para os trabalhadores da área assistencial em comparação com aqueles de ocupações administrativas.

São três os principais personagens envolvidos nas questões de relacionamento social: colegas de trabalho, chefias e usuários. Nesse sentido, é importante considerar que a área assistencial tende a apresentar um quantitativo mais expressivo desses personagens, já que atende grande número de usuários e, em razão do regime de escalas e da existência de equipes multiprofissionais, há maior rotatividade de chefias e colegas de trabalho $^{14}$. Uma boa QVT passa pela satisfação dos trabalhadores em encontrar-se num ambiente seguro, de respeito mútuo e com oportunidades para o desempenho de suas funções ${ }^{20}$.

Em relação às oportunidades de crescimento profissional, a avaliação da área administrativa $(4,21 \pm 3,39 ; \mathrm{p}=0,006)$ é significantemente inferior à da área médica $(6,50 \pm 2,02 ; \mathrm{p}=0,006)$. $\mathrm{Em}$ diferentes itens sobre reconhecimento do trabalho, são os funcionários da área assistencial que apresentam escores significativamente inferiores aos da área médica.

Existem modalidades de reconhecimento e crescimento profissional normalmente evocadas pelos trabalhadores, que são: retribuição monetária e prática do elogio ${ }^{14}$. Diante dos resultados obtidos, é importante discutir quais seriam as possíveis lacunas no âmbito da instituição.

Nesse âmbito, mostra-se salutar que, na política de QVT, estejam contempladas oportunidades para o crescimento na carreira e, ainda, a possibilidade de que os trabalhadores participem de processos de tomada de decisão, independen- temente de sua área de atuação, o que resultará em um melhor reconhecimento e maior satisfação dos trabalhadores da empresa ${ }^{1,20}$.

Os escores mais elevados foram registrados para a área médica, sendo importante atentar para várias questões que podem envolver certa diferenciação entre as categorias (jornada, condições e organização de trabalho, valorização diferenciada pela comunidade e pelos gestores, etc.).

Em contrapartida, os profissionais administrativos revelam um nível maior de mal-estar no trabalho, relacionado, sobretudo, ao crescimento profissional. Algumas reflexões, então, se apresentam. É possível que esses trabalhadores se sintam em ocupações aquém de suas potencialidades ou que o fato de a assistência à saúde ser a atividade principal da instituição tenha impacto motivacional negativo, levando-os a crer que as realizações administrativas estão em segundo plano.

$\mathrm{Na}$ análise sobre o Elo Trabalho-Vida Social, os trabalhadores da área administrativa também apresentaram uma percepção da utilidade do trabalho para a sociedade significativamente inferior $(8,82 \pm 1,68 ; p=0,007)$ em comparação com os da área assistencial $(9,48 \pm 0,80 ; p=0,007)$ e médica $(9,63 \pm 0,88 ; p=0,004)$. Ferreira ${ }^{14}$ afirma que o trabalho produtor de felicidade está também intimamente imbricado com a noção de utilidade que o labor representa.

As descobertas do estudo apontam para a necessidade de concentrar ações de atenção à QVT nos trabalhadores das áreas assistencial e administrativa, dependendo do fator estruturante contemplado nas atividades. As grandes diferenças encontradas entre as áreas servem de alerta para a complexidade da gestão da QVT. Em análise geral, Souza ${ }^{4}$ afirma que seria mais relevante se as organizações visualizassem QVT como um direito à saúde do trabalhador, e não como ferramenta para melhorar a produtividade.

As intervenções direcionadas que levam em conta diferenças de gênero, emprego, setoriais e regionais tendem a ter maior impacto nos resultados de saúde ${ }^{31}$. Portanto, um programa de caráter preventivo e que atue na resolutividade das situações-problemas se configura em grande oportunidade de amenizar a insatisfação nas relações de trabalho.

Foi identificado como limitação da pesquisa o desenho epidemiológico empregado. Com o estudo transversal foi necessário apoiar-se exclusivamente da literatura explorada para buscar uma direção causal das evidências encontradas. 


\section{Conclusão}

Foram evidenciadas diferenças importantes na percepção de QVT quando comparamos trabalhadores de áreas profissionais distintas, fornecendo fortes evidências de que um planejamento eficiente de QVT, que pode resultar em promoção da saúde dos trabalhadores, necessita atender demandas setorizadas.

Em sua maioria, os autores que abordam o tema deixam de considerar a relevância da estruturação setorizada para o sucesso da atenção à QVT. Nossas descobertas mostram que em uma instituição hospitalar trabalhadores da área assistencial apresentam QVT inferior àqueles das áreas administrativa e médica. $\mathrm{E}$ a área administrati- va também evidencia QVT inferior à área médica. Esses achados enfatizam a necessidade de as intervenções no local de trabalho serem inclusivas e de atingir os trabalhadores com maior dificuldade.

Os resultados deste estudo podem contribuir para que a fase de diagnóstico de programas de QVT comtemple análises com diferenciação entre grupos com atuações profissionais distintas. Esta pesquisa sugere que, ao se planejar intervenções no local de trabalho, pode ser benéfico direcionar as ações em função das necessidades dos diferentes conjuntos de trabalhadores que integram uma instituição. Tratar grupos diferentes de maneira específica parece ser um alicerce seguro para a construção de uma QVT capaz de promover a saúde.

\section{Colaboradores}

SF Camargo trabalhou na concepção do projeto, metodologia, coordenação da pesquisa e redação final. RHSC Almino trabalhou na concepção do projeto, pesquisa, metodologia e redação. MP Diógenes trabalhou na concepção do projeto e pesquisa. JP Oliveira Neto trabalhou na pesquisa. IDS Silva trabalhou na pesquisa. LC Medeiros trabalhou na pesquisa. KGR Dantas trabalhou na redação. JDAS Camargo trabalhou na orientação do projeto, concepção, metodologia, análise estatística e redação. 


\section{Referências}

1. Nanjundeswaraswamy TS, Swamy DR. Review of literature on quality of worklife. Int J Qual Res 2013; 7:201-214

2. Silva CA, Ferreira MC. Dimensões e Indicadores da Qualidade de Vida e do Bem-Estar no Trabalho. Psicol Teor Pesqui 2013; 29:331-339.

3. Antloga CS, Maia M, Cunha KR, Peixoto J. Contexto de trabalho e custo humano no trabalho em um órgão do Poder Judiciário brasileiro. Cien Saude Colet 2014; 19(12):4787-4796

4. Souza EBDE. Competitividade empresarial, gestão de pessoas e controle social : para pensar os dilemas da Qualidade de Vida no Trabalho (QVT). Rev Espaço Acadêmico 2015; 172:112-123.

5. Barbosa FLS, Bizarria FPDA, Rabêlo Neto A, Moreira RN. Visão multidimensional da satisfação do trabalho: um estudo em um hospital público piauiense. REGE 2016; 23(2):99-110.

6. Grote $G$, Guest D. The case for reinvigorating quality of working life research. Hum Relations 2017; 70:149167.

7. De Sio S, Cedrone F, Sanità D, Ricci P, Corbosiero P, Di Traglia M, Greco E, Stansfeld S. Quality of Life in Workers and Stress: Gender Differences in Exposure to Psychosocial Risks and Perceived Well-Being. Biomed Res Int 2017; 2017:7340781.

8. Barbosa ML, Menezes TN, Santos SR, Olinda RA, Costa GMC. Qualidade de vida no trabalho dos profissionais de saúde no sistema prisional. Cien Saude Colet 2018; 23(4):1293-1302.

9. Ferreira MC. Qualidade de Vida no Trabalho (QVT): do assistencialismo à promoção efetiva. Laboreal 2015; 11:28-35.

10. Pizzio A, Klein K. Qualidade de vida no trabalho e adoecimento no cotidiano de docentes do Ensino Superior. Educ Soc 2015; 36:493-513.

11. Fernandes LC, Ferreira MC. Qualidade de vida no trabalho e risco de adoecimento: estudo no poder judiciário brasileiro. Psicol USP 2015; 26:296-306.

12. Mascarenhas CHM, Prado FO, Fernandes MH. Factors associated with the quality of life of community health agents. Cien Saude Colet 2013; 18(5):13751386.

13. Brasil. Ministério da Saúde (MS). As cartas da promoção da saúde. Brasília: MS; 2013.

14. Ferreira MC. Qualidade de vida no trabalho: uma abordagem centrada no olhar dos trabalhadores. Brasília: LPA; 2011.

15. Valero Pacheco IC, Riaño-Casallas MI. Contributions of occupational health and safety to the quality of working life: An analytical reflection. Contrib Salud Segur Trab Calid Vida Labor Reflexión Analítica 2017; 15:85-94.

16. Ferreira MC, Brusiquese RG. Novas condições de trabalho e velhos modos de gestão: A qualidade de vida no trabalho em questão. Rev Bras Gest Desenvolv Reg 2014; 10:247-267.

17. Hipólito MCV, Masson VA, Monteiro MI, Gutierrez GL. Qualidade de vida no trabalho: avaliação de estudos de intervenção. Rev Bras Enferm 2017; 70(1):189-197.

18. Dilig-ruiz A, Macdonald I, Varin MD, Vandyk A, Graham ID, Squires JE. Job satisfaction among critical care nurses : A systematic review. Int J Nurs Stud 2018; 88:123-134.
19. Fernandes JDC, Portela LF, Griep RH, Rotenberg L. Jornada de trabalho e saúde em enfermeiros de hospitais públicos segundo o gênero. Rev Saude Publica 2017; 51:63.

20. Daubermann DC, Tonete VLP. Qualidade de vida no trabalho do enfermeiro da Atenção Básica à Saúde. ACTA Paul Enferm 2012; 25:277-283.

21. Silva AA, Rotenberg L, Fischer FM. Jornadas de trabalho na enfermagem: entre necessidades individuais e condições de trabalho. Rev Saude Publica 2011; 45:1117-1126.

22. Lima DMG, Araújo RC, Pitangui ACR, Rizzo J, Sarinho S, Santos C, Costa E, Cerreia Junior M. Descrição da atividade física e da jornada de trabalho na qualidade de vida de profissionais de terapia intensiva: Comparação entre um grande centro urbano e uma cidade do interior brasileiro. Rev Bras Atividade Física Saúde 2015; 20(4):386-396.

23. Olivares A, Bonito J, Silva R. Qualidade de vida no trabalho dos médicos da atenção básica no estado de Roraima (Brasil). Psicol Saúde Doença 2015; 16:100-111.

24. Azevedo WF, Mathias LAST. Adição ao trabalho e qualidade de vida: um estudo com médicos. Einstein 2017; 15:130-135.

25. Fabichak C, Silva-Junior JS, Morrone LC. Síndrome de burnout em médicos residentes e preditores organizacionais do trabalho. Rev Bras Med Trab 2014; 12:79-84.

26. Ferreira MC, Alves L, Tostes N. Gestão da qualidade de vida no trabalho (QVT) no serviço público federal: o descompasso entre problemas e práticas gerenciais. Psicol Teor Pesqui 2009; 25:319-327.

27. Silva UL, Oliveira AF. Qualidade de Vida e Valores nas Organizações: Impactos na Confiança do Empregado. Psicol Cien Profis 2017; 37:7-17.

28. Anderson DR, Sweeney DJ, Williams TA. Estatística aplicada à administração e economia. $2^{\text {a }}$ ed. São Paulo: Cengage Learning; 2011.

29. Ferreira MC. Inventário de avaliação de qualidade de vida no trabalho (IA_QVT): instrumento de diagnóstico e monitoramento de QVT nas organizações. Anais 61 Reun Anu Soc Bras Prog Cien (SBPC) 2009.

30. Brunault P, Fouquereau E, Colombat P, Gillet N, El -Hage W, Camus V, Gaillard P. Do Transactive Memory and Participative Teamwork Improve Nurses' Quality of Work Life? West J Nurs Res 2014; 36(3):329345.

31. Kazi A, Haslam C, Duncan M, Clemes S, Twumasi R. Sedentary behaviour and health at work: an investigation of industrial sector, job role, gender and geographical differences. Ergonomics 2018; 62(1):21-30.

Artigo apresentado em 11/07/2018

Aprovado em 05/06/2019

Versão final apresentada em 07/06/2019

Editores-chefes: Romeu Gomes, Antônio Augusto Moura da Silva 